\title{
Biofilm integrity and cytomorphology of Candida albicans after exposure to UV-light on ZnO thin films: SEM Analysis
}

Carlos Arzate-Quintana ${ }^{1}$, César Leyva-Porras ${ }^{2}$, María Alejandra Favila-Pérez ${ }^{1}$, Alva Rocío CastilloGonzález $^{1}$, Celia María Quiñonez-Flores ${ }^{1}$ and Alejandro Faudoa-Arzate ${ }^{3}$

${ }^{1}$ Facultad de Medicina y Ciencias Biomédicas de la Universidad Autónoma de Chihuahua, Chihuahua, Chihuahua, Mexico, ${ }^{2}$ Centro de investigación en materiales avanzados (CIMAV), Chihuahua, Chihuahua, Mexico, ${ }^{3}$ School of Engineering and Sciences, Tecnológico de Monterrey, Chihuahua, Chihuahua, Mexico

\section{Introduction}

Candida albicans is an opportunistic pathogen known as a potential source of infection in the hospital environment [3]. Besides antimicrobial resistance, this microorganism has different pathogenic factors that allow survival on surfaces as well as in organic tissues, and part of this mechanism is the ability of changing from a yeast cell to hyphae and pseudohyphae structures [3, 6]. Intrahospital infections of Candida albicans are reported to have a mortality rate in immunocompromised patients of 40 to $50 \%$ [3, 5] and this figure can increase to $72 \%$ in countries with less efficient healthcare systems [7], furthermore, new species of Candida are being reported to emerge as new pathogens in this group of patients [4]. Because of the impact of hospital adapted pathogens in the health care systems, the development of new strategies of disinfection to prevent the spread of infections should be considered top priority. A suggested strategy is the use of photocatalytic coatings consisting in metal oxides, which are being tested as potential antimicrobial technology [1] with promising results. The present work is focused in the analysis of the effect of UV-light to disinfect Zinc Oxide $(\mathrm{ZnO})$ thin films inoculated with $C$. albicans from the SEM analysis perspective.

\section{Materials and Methods}

$\mathrm{ZnO}$ thin films were synthesized by the spray pyrolysis method by addition of Zinc acetate to a $80^{\circ} \mathrm{C}$ heated glass surface (slides). Thin films characterization was performed by X-Ray diffraction, SEM (JSM5800LV) microscopy and EDS (data not shown). Candida albicans ATCC 10231 was maintained in Brain-Heart infusion until use in antimicrobial assays. A $10 \mu 1$ drop of sterile distilled water containing $1.6 \times 10^{7}$ yeast $/ \mathrm{ml}$ was deposited in $\mathrm{ZnO}$ thin films, and then separated into two groups: a control group with no exposure to UV light, a group exposed to 60 minutes to $254 \mathrm{~nm}$ UV light. ZnO thin films were then stored 14 days at room temperature until SEM analysis.

\section{Results}

As reported in previous publications, UV radiation decreases the number of yeast on the surface of photocatalytic thin films [1] and, as it can be observed in 500X micrographs (Figure 1), the number of cells is lower after 60 minutes of exposure. It can also be noted that the morphology of yeast tends to change to pseudohyphae after the treatment with UV light, this result was expected since genetic stress is known to promote the appearance of pseudohyphae, and ionizing radiation is a direct cause of genotoxicity [2]. This kind of morphology changes are relevant since they are an important virulence factor of various pathogenic candida species [6]. 
Analysis of 2,500X micrographs was realized by the backscattered electrons technique with the aim to promote a clear contrast between the organic matter (dark grey) and $\mathrm{ZnO}$ thin films (brilliant white), an effect obtained thanks to the difference in atomic weight. Figure 2-A shows organic matter surrounding yeast cells not exposed to UV light, presumably this structure is an important pathogenic mechanism known as "biofilm". This result is consistent with reported literature, since yeasts cells tend to produce biofilms that protect fungal cells from adverse environments, disinfectants and antimicrobials [5, 7]. These biofilms are not observable in $\mathrm{ZnO}$ films that were exposed to UV light for 60 minutes (Figure 2-B), meaning that this disinfection method not only eliminates living cells, but it also makes $C$. albicans more susceptible to other disinfection treatments.

Cytomorphology of yeast cells was studied in micrographs at 10,000X, biofilms difficult the comparison between cells before and after treatments (Data not shown), nevertheless there is not an important difference between cell structure on both images.
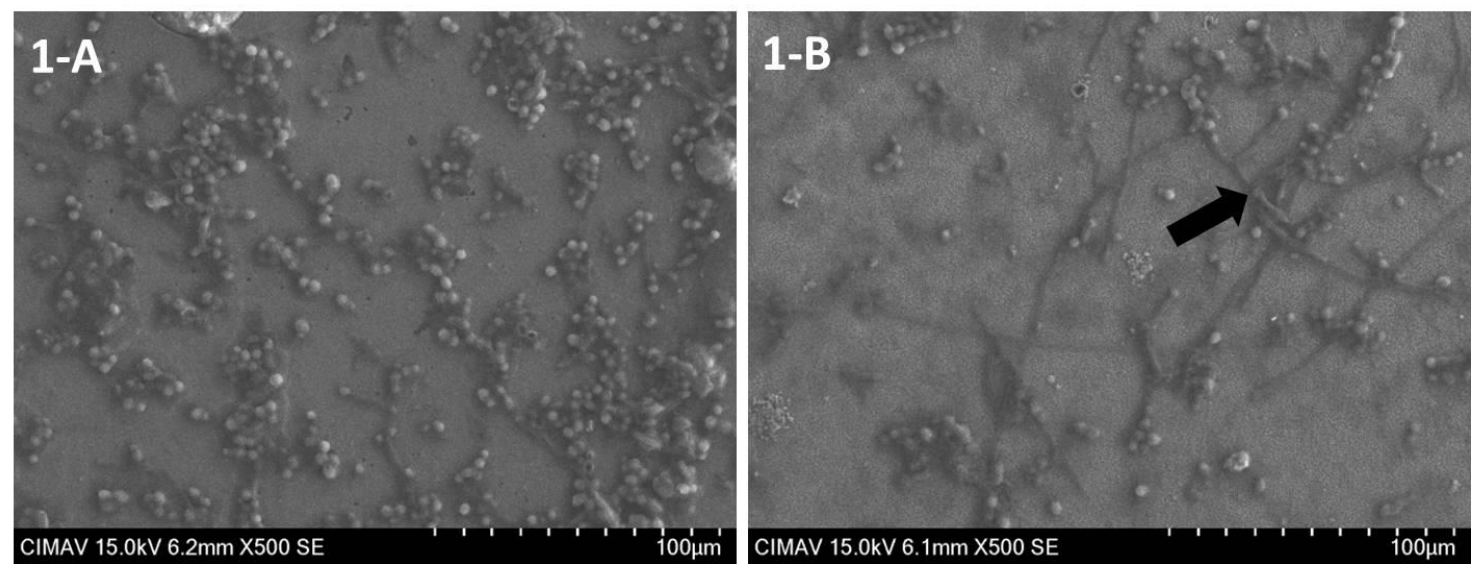

Figure 1. Comparison between $\mathrm{ZnO}$ films inoculated with $\mathrm{C}$. albicans at 500X augments. 1-A consist in a control not exposed to UV-light. Micrograph 1-B shows a film exposed to UV-light for 60 minutes, lower quantities of cells can be observed, however, as highlighted by the black arrow, the number of pseudohyphae increased.
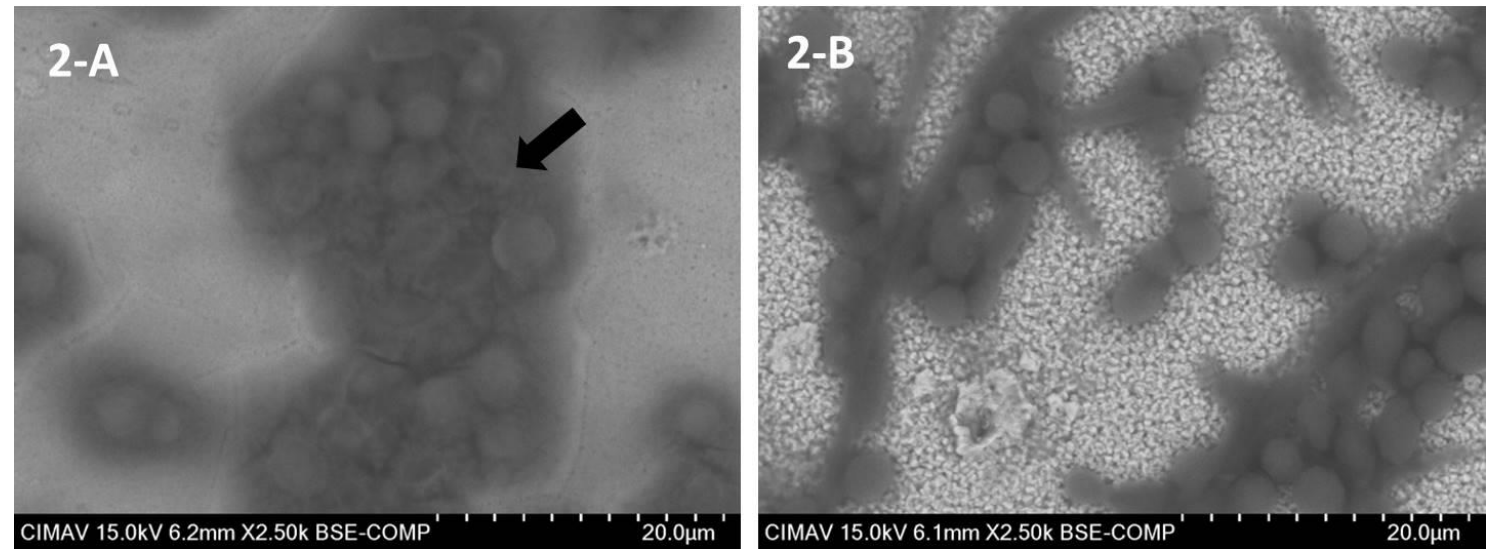

Figure 2. Comparison of $\mathrm{ZnO}$ thin films inoculated with $\mathrm{C}$. albicans at 2,500X augments using backscattered electrons technique. Micrograph 2-A consists of a control not exposed to UV-light, in this sample the presence of biofilms can be observed surrounding yeast cells (highlighted by black arrow), 2B shows a sample exposed to UV-light during 60 minutes; the biofilm is no longer observable. 
References

[1] Arzate-Quintana C, Camarillo-Cisneros J, Realyvazquez-Guevara P, Faudoa-Arzate A, \& Rodríguez H. 2020. SEM Study of the Photocatalytic Activity of SnO2 Films Exposed to UV Radiation Against the Human Pathogen C. albicans. Microscopy and Microanalysis, Vol. 26(S2), pp. 1362-1365. doi:10.1017/S1431927620017845.

[2] Bravo Ruiz G, Ross ZK, Gow NAR, Lorenz A. 2020. Pseudohyphal growth of the emerging pathogen Candida auris is triggered by genotoxic stress through the $\mathbf{S}$ phase checkpoint. $m S p h e r e$ Vol 5:e0151-20, pp 1-17. https://doi.org/10.1128/mSphere.00151-20.

[3] Chen H, Zhou X, Ren B \& Cheng L. (2020). The regulation of hyphae growth in Candida albicans. Virulence, Vol. 11:1, pp. 337-348, DOI: 10.1080/21505594.2020.1748930.

[4] Fakhim H, Vaezi A, Dannaoui E, Chowdhary A, Nasiry D, Faeli L, Meis JF \& Badali H. Comparative virulence of Candida auris with Candida haemulonii, Candida glabrata and Candida albicans in a murine model. Mycoses, Vol. 61: pp. 377-382. DOI: 10.1111/myc.12754.

[5] Kornitzer D. 2019. Regulation of Candida albicans Hyphal Morphogenesis by Endogenous Signals. J. Fungi, Vol. 5, 21; doi:10.3390/jof5010021.

[6] Mukaremera L, Lee KK, Mora-Montes HM\& Gow NAR. 2017. Candida albicans Yeast, Pseudohyphal, and Hyphal Morphogenesis Differentially Affects Immune Recognition. Front. Immunol. Vol. 8, pp 629. https://doi.org/10.3389/fimmu.2017.00629.

[7] Sharma J, Rosiana S, Razzaq I \& Shapiro RS. 2019. Linking Cellular Morphogenesis with Antifungal Treatment and Susceptibility in Candida Pathogens. J. Fungi, Vol. 5, 17; doi:10.3390/jof5010017. 